\title{
Relación terapéutica
}

$\mathrm{L}$ a enfermería, al igual que las otras ciencias, ha sufrido diversos cambios a lo largo de la historia, cada uno de los cuales obedece a un contexto histórico determinado que permite comprender la evolución y lo que ha sucedido alrededor de ella (1). Sin embargo, la enfermería ha mantenido un rasgo común a lo largo de la historia, la acción de cuidar, y su quehacer se ha centrado siempre en un servicio de cuidado a los otros, realizado de maneras muy distintas

\section{Breve historia de la acción de cuidar}

a) Primer momento: enfermería religiosa.

Acontece cuando la acción de cuidar estaba vinculada a la profesión religiosa; tal era el caso de la asistencia hospitalaria en los monasterios, lazaretos, refugios de peregrinos, o de una de las más decisivas influencias que ha tenido la enfermería en la historia: las Hijas de la Caridad, fundadas por San Vicente de Paúl.

b) Segundo momento: secularización de la enfermería o modelo clásico.

Esta dimensión aparece cuando se seculariza la enfermería y la profesionalización empieza a gestarse con la llamada matriarca de la enfermería, Florence Nightingale (1820-1910). Fue ella la que estableció varias instituciones de enseñanza para enfermeras, a quienes dotaba de una sólida preparación teórica y técnica. Sin embargo, la vinculación religiosa era clara: la misma Florence animaba a sus súbditas a "hacer la obra de Dios en este mundo, con corazón limpio y sencillo" (1).

El modelo clásico de enfermería entiende el cuidado como aquella virtud que debe llevar a cabo la (el) enfermera (o) profesional, que consiste en "colocar al paciente en las mejores condiciones para que la naturaleza actúe sobre él". Una (un) enfermera(o) profesional no medica antes bien "cultiva todo aquello que conduce a la observación... que es justo el camino para acabar con las aficionadas para hacer de médicos, y si los médicos les obligaran a obedecerles, ellas les ayudarían, en lugar de servirles de estorbo" (2). c) Emancipación de la enfermería

Un salto en la historia de la enfermería se da en las décadas de los años 60 y 70, con una imagen de la (el) enfermera (o) que se autodenomina abogada o defensora del paciente. Para esta(e) enfermera(o), los cuidados consisten en proteger y defender los derechos del paciente, además de prepararlo física y psicológicamente para que se defienda de las agresiones de médicos y estructuras sanitarias.

Bajo esta perspectiva, la autonomía se reivindica sobremanera, tanto en la (el) enfermera(o) que defiende su profesión como soberana e independiente, como en el paciente que se percibe amenazado en un ambiente hospitalario hostil que menoscaba sus derechos, modelo muy propio de los Estados Unidos. En síntesis, para dar buenos cuidados ha de ser leal e incondicional, no al médico sino al paciente, y se ha de destacar por la máxima independencia profesional.

d) Enfermería como una profesión autónoma desde la compasión y el cuidado.

Nunca como en los últimos quince años se ha investigado tanto en el contenido de los cuidados de enfermería, buscando desentrañar lo que implica el cuidar. La enfermería requiere actuar de manera que preserve la dignidad humana, restaure la humanidad y evite reducir personas al estatuto moral de objetos. Para Kristen Swanson, de acuerdo con Páez (1), el cuidado requiere específicamente:

1. Conocimiento o búsqueda para entender un hecho con su significado tal cual en la vida del otro.

2. Estar con el otro, es decir, estar emocionalmente presente con el otro.

3. Hacer por el otro, es decir, hacer cuanto el sujeto haría por sí mismo(a) si le fuera posible.

4. Ayudar a/o facilitar el paso del otro por transiciones de la vida o por situaciones desconocidas.

5. Mantener la fe, que se refiere a sostener la fe en la capacidad del otro de salir adelante a través de un hecho o transición, y dar la cara a un futuro pleno.

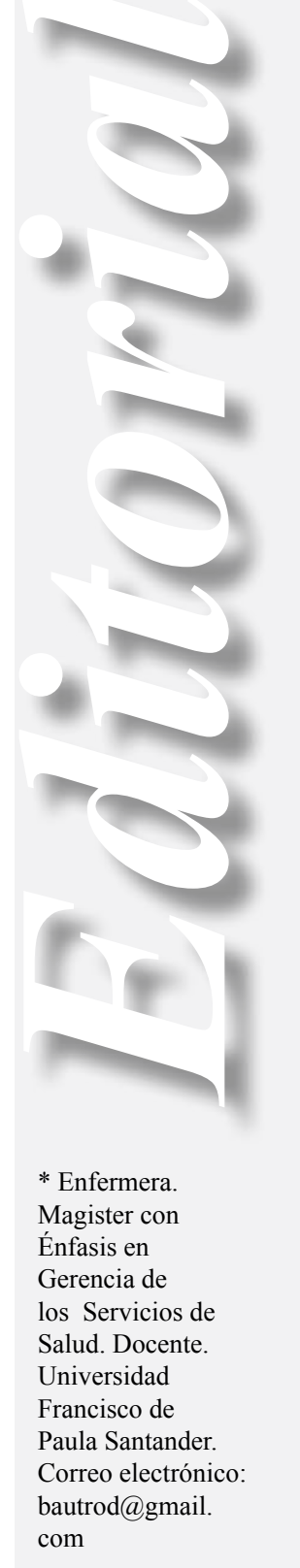


El acto de cuidar es propio de la naturaleza humana, de modo que todo ser humano es capaz de cuidar de sí y de otro. El objetivo del trabajo de enfermería es el cuidado, premisa que ha sido aceptada por los profesionales, razón por la cual, cuando se define enfermería, se dice que es la ciencia y el arte del cuidado.

\section{Modelos y teorías que fundamentan la relación terapéutica}

El cuidado del individuo, la familia y la comunidad constituyen el centro de atención y el deber ineludible de cualquier profesional de enfermería. La calidad de este cuidado requiere de personas con habilidades interpersonales que favorezcan el ofrecimiento de cuidados diferenciales y significativos para quien los recibe.

La relación enfermera(o)-paciente es un aspecto muy importante para desarrollar los cuidados de enfermería. La relación terapéutica personaenfermera se constituye en la base para poder brindar los cuidados de enfermería y, para lograrlo, es necesario establecer una relación basada en la confianza como requisito indispensable para la buena marcha del proceso terapéutico. No sólo el enfermo debe confiar en la (el) enfermera(o), sino también ésta(e) debe confiar en sí misma(o) como terapeuta, en la motivación del paciente y las capacidades para su cambio.

La confianza es la base fundamental de una relación terapéutica; la consistencia y establecimiento de límites se constituyen en los elementos claves para generarla. Los límites efectivos proporcionan sentido a la relación establecida y reducen al mínimo la manipulación del paciente.

En la evolución histórica de la disciplina de enfermería, la relación persona-enfermera(o) aparece como una constante que ha sido abordada por algunas investigadoras de la profesión que definen enfermería como una relación que ayuda, que acompaña a la persona, dentro de un entorno, a vivir experiencias de salud. Los conceptos teóricos que fundamentan la relación de ayuda fueron desarrollados, según Hernández y Flores (3), por estudiosos del tema como Hildegarde Peplau, Jean Orlando y Joice Travelbee.

Peplau, en su Modelo de Enfermería Psicodinámica, aborda un tipo de relación enfermera(o)-paciente que se conoce como relaciones interpersonales, en el cual dos personas llegan a conocerse lo suficientemente bien como para afrontar los problemas que surjan de forma cooperativa (3).

Según este autor, las intervenciones de las enfermeras son procesos interpersonales terapéuticos y significativos para la persona. Los cuidados han de ser individualizados, haciendo especial hincapié en la relación humana entre la (el) enfermera(o) y la persona necesitada de ayuda.

Peplau, de acuerdo con Marriner y Raile (4), describe cuatro fases en la relación enfermera(o)-paciente:

- Orientación: el individuo presenta una necesidad sentida y busca asistencia profesional. El enfermero(a) ayuda al paciente tanto a reconocer y comprender su problema como a determinar qué tipo de ayuda necesita.

- Identificación: el paciente se identifica con aquellos que pueden ayudarle (relación).

- Explotación: el paciente trata de obtener el mayor beneficio de todo lo que se le ofrece a través de la relación.

- Resolución: se produce cuando se abandonan, de forma gradual, los viejos objetivos y se adoptan otros nuevos.

Orlando, en su teoría del proceso deliberativo, centra su teoría en la relación de reciprocidad entre la enfermera y el paciente. "Lo que uno hace o dice, afecta al otro o viceversa" (3). Destaca la importancia de explorar las percepciones, los sentimientos y los pensamientos y de someter dicha indagación a la verificación de la otra parte. Esta disciplina de proceso o exploración da cuenta de lo que ella denomina necesidades de ayuda por parte del enfermo.

Considera que la función de enfermería es averiguar y satisfacer las necesidades de ayuda inmediata al paciente, la cual se logra cuando la enfermera satisface estas necesidades a través del proceso de enfermería.

Travelbee, en su modelo de persona a persona, afirma que el propósito de la enfermería se alcanza mediante el establecimiento de una relación a través de interacciones de humano a humano; la cual se origina en el encuentro inicial con la identificación, desarrollo de sentimientos de empatía, solidaridad y afinidad. A medida que el proceso de interacción progresa hacia la relación de afinidad, se obtiene el potencial necesario para una relación terapéutica (3). 
Los principales conceptos que integran este modelo son:

a) Comunicación: proceso por el cual el enfermero(a) es capaz de establecer una relación de ayuda interpersonal con el paciente y su familia para enfrentar la experiencia de la enfermedad y el sufrimiento.

b) Interacción: se refiere a cualquier contacto entre dos individuos durante el cual se ejerce una influencia recíproca y, entre sí, una comunicación verbal o no verbal.

c) Empatía: la capacidad de comprender el estado psicológico y emocional del otro.

d) Simpatía: implica el deseo de ayudar al individuo que se encuentra sometido a estrés.

e) Relación de persona a persona: es primariamente una experiencia o una serie de experiencias que se dan entre el enfermero(a) y el receptor de sus cuidados, con el propósito de satisfacer las necesidades de cuidado de enfermería presentes en los individuos, familias y comunidades, para prevenir o enfrentar la experiencia que supone la enfermedad $\mathrm{y}$, si es necesario, encontrarle un sentido a dichas experiencias.

\section{La relación terapéutica en un servicio de hospitalización}

En la hospitalización hay un cambio de entorno que exige una adaptación. Un sobreesfuerzo por parte de la persona que ingresa, ya que no sólo debe desear su curación sino que debe adaptarse a un entorno diferente al de su cotidianidad, con unas normas nuevas y donde prima la generalidad sobre la individualidad. Así, el sujeto vive el ingreso hospitalario como una situación de estrés, ansiedad y vulnerabilidad.

En el proceso de admisión a un hospital, algunos pacientes no aceptan que sus roles han cambiado y se comportan de una manera distinta, siendo sistemáticamente tachados por los profesionales como pacientes difíciles o problemáticos, estigma que, por ser fundamental, afecta la relación personaenfermera(o).

Entre los distintos factores que determinan la relación enfermera(o)-persona en el medio hospitalario, la falta de tiempo es el más verbalizado entre las profesionales. Sin embargo, se cree que la falta de técnica y de formación sobre la relación terapéutica es el factor más determinante. Para situar la relación terapéutica es preciso considerar tres conceptos básicos: enfermería, entorno y relación (5).

Figura 1. Marco conceptual de la relación enfermero(a)-paciente.

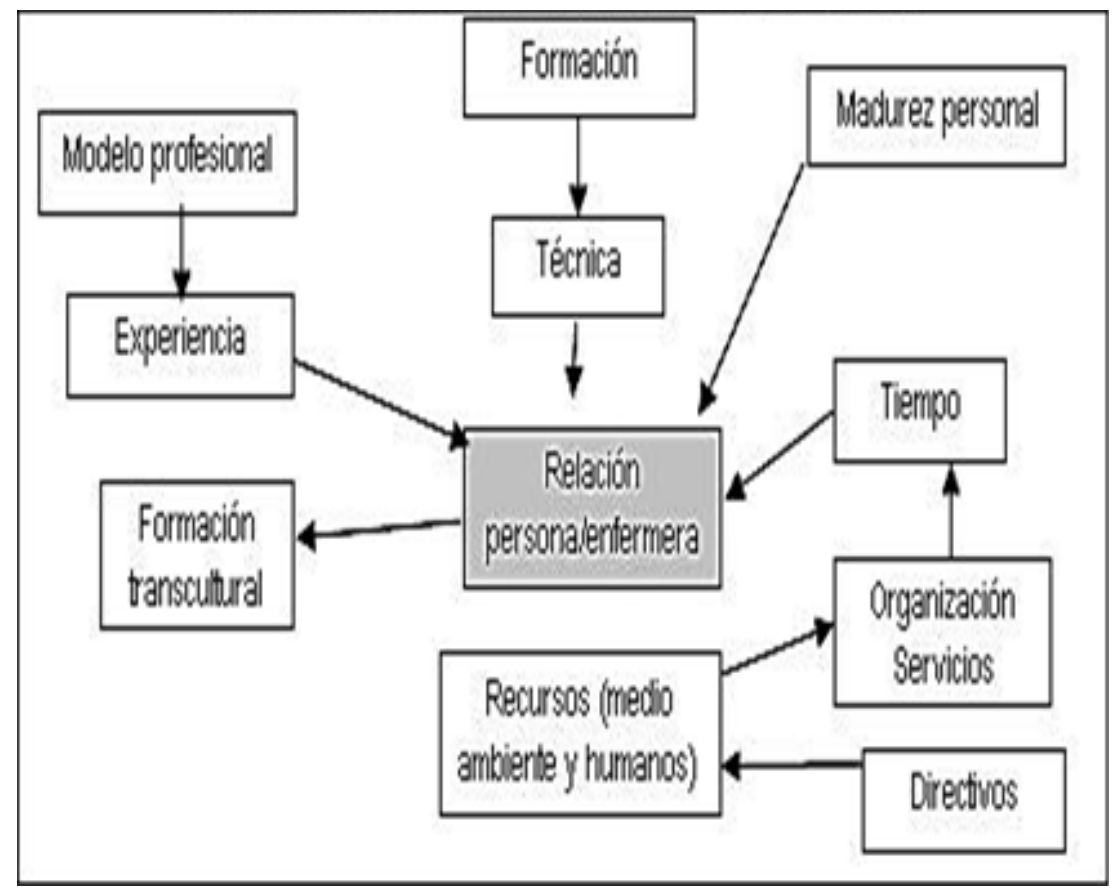

Fuente: Tomado y adaptado de Stuart \& Sundeen, 1995. 


\section{Enfermería}

Peplau definió enfermería como "un importante proceso terapéutico interpersonal que actúa en coordinación con otros procesos humanos que hacen posible la salud de los individuos en las comunidades (4).

Orlando considera, desde otra mirada, que la enfermería es una profesión independiente que funciona de una manera autónoma, ofreciendo una asistencia directa a las personas en cualquier situación en que se encuentren, con el ánimo de evitar, aliviar, reducir o curar su sensación de impotencia (4).

Travelbee, a su vez, definió enfermería como un proceso interpersonal por el cual el profesional de enfermería ayuda a una persona, una familia o una comunidad a prevenir o afrontar la experiencia de la enfermedad y el sufrimiento y, en caso necesario, a dar sentido a estas experiencias (4).

\section{Entorno}

El entorno es principalmente exterior a la persona, pero ejerce una influencia en lo cotidiano y en la existencia de esta persona. Peplau lo define entorno como aquellas fuerzas existentes fuera del organismo y en el contexto de cultural.
Orlando, por el contrario, no define el entorno y asume que la situación de enfermería se da cuando existe un contacto entre enfermero(a) y paciente y tanto uno como otro perciben, piensan, sienten y actúan en la situación inmediata. Destaca que un paciente puede reaccionar sufriendo ante cualquier aspecto de un entorno que ha sido diseñado con fines terapéuticos y de ayuda (4).

En la misma dirección, Travelbee tampoco define explícitamente el término entorno en la situación de enfermería y opta por otros aspectos como la condición humana y las experiencias vitales por las que atraviesan todos los seres humanos, como el sufrimiento, la esperanza, el dolor y la enfermedad (4).

\section{Relación}

En este contexto, la relación se entiende como el encuentro terapéutico entre enfermera(o)-persona guiado hacia un objetivo común que es la vivencia de experiencias de salud. En esta relación hay elementos que pueden modificarla, cambiarla, afectarla o potenciarla como se puede observar en la figura 2 (5).

Figura 2. Elementos que afectan la habilidad de las enfermeras para ser terapéuticos con los pacientes.

\begin{tabular}{|c|c|c|}
\hline $\begin{array}{l}\text { Cualidades } \\
\text { personales }\end{array}$ & $\Rightarrow$ & $\begin{array}{l}\text { Autoconocimiento, Valores claros, } \\
\text { Exploración de sentimientos, } \\
\text { Modelos de Rol, Altruismo, Ética y } \\
\text { responsabilidad }\end{array}$ \\
\hline \multicolumn{3}{|r|}{ 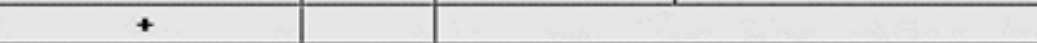 } \\
\hline $\begin{array}{l}\text { Facilidad para la } \\
\text { comunicación }\end{array}$ & $\Rightarrow$ & $\begin{array}{l}\text { Comportamiento verbal, } \\
\text { Comportamiento no verbal, Análisis } \\
\text { de problemas, Técnicas terapéuticas }\end{array}$ \\
\hline \multicolumn{3}{|r|}{ 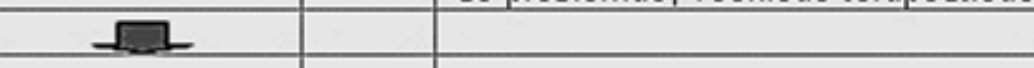 } \\
\hline Respuestas & $\Rightarrow$ & Genuina, Respeto, Empatía \\
\hline \multicolumn{3}{|l|}{+} \\
\hline Acciones & $\Rightarrow$ & $\begin{array}{l}\text { Confrontación, Darse a conocerse, } \\
\text { Catarsis, Role playing, Inmediatas }\end{array}$ \\
\hline $\begin{array}{l}\text { Dificultades } \\
\text { terapéuticas } \\
\end{array}$ & $\Rightarrow$ & $\begin{array}{c}\text { Resistencia, Relaciones de } \\
\text { transferencia, Violación de los límites } \\
\text { terapéuticos }\end{array}$ \\
\hline $\begin{array}{l}\text { Resultado } \\
\text { terapéutico }\end{array}$ & $\Rightarrow$ & $\begin{array}{c}\text { Para el paciente, Para la sociedad, } \\
\text { Para la enfermera }\end{array}$ \\
\hline
\end{tabular}

Fuente: Tomado y adaptado de Stuart \& Sundeen, 1995. 
Cuando cualquier enfermera o enfermero de hospitalización inicia su jornada laboral, no hay planteamiento sobre qué cuido o cómo cuido, sólo la rutina de la tarea por hacer y cumplir una jornada laboral.

\section{Dos enfoques del cuidado profesional (5)}

En la profesión de enfermería, según Cibanal y Arce (6), hay dos maneras de enfocar los cuidados de enfermería, que son dos abordajes fundamentales:

1. Abordaje subjetivo científico, bien reconocido, utilizado y valorizado por todos. En él la enfermera da cuidados del mundo exterior, utiliza un plan, un protocolo preciso e identifica lo más objetivamente posible los signos, síntomas, los problemas y las necesidades del paciente (está fuera de ella).

Aquí la (el) enfermera(o) tiene un rol de cuidador(a) no muy bien identificado pero suficiente para ser reconocido en la sociedad, aunque no siempre se siente bien en el papel que ésta le otorga. Por otra parte, cuando la salud física está bien separada de la salud psicológica, la situación de la (el) enfermera(o) es confortable en cuanto a los cuidados o las técnicas que esta(e) profesional imparte. Son actos legales, cuantificables, recopilados y defendibles

Este modelo coincide con el rol profesional reconocido por la sociedad. Actualmente, dentro de la generalidad, se ha aceptado trabajar siempre con funciones dependientes y delegadas, desempeñándose con toda rapidez y con el consiguiente riesgo de la pérdida de atractivo por los cuidados propios de enfermería y de la profesión, lo posteriormente se denominó como síndrome de Burn-Out, muy frecuente entre las enfermeras.

De esta forma, Cibanal y Arce (6) manifiestan que aquel profesional que no está centrado en su identidad no llega a encontrar valor en todo lo que hace y en la evaluación de los otros; se dispersa en toda clase de tareas, se confunde con los otros y con su servicio a los otros, lo cual lo lleva a no sentirse realizado.

2. Abordaje inter-subjetivo, más global y profundo, ya que la (el) enfermera(o) debe integrar relación, proceso de cuidados y modelo de enfermería. Esta integración es un proceso largo y exige una implicación profesional y personal; no es un proceso cuantificable y no es registrado.

\section{Algunos problemas de la relación terapéutica (5)}

En este contexto de falta de identidad, siempre se defiende que la enfermería es la profesión que más tiempo pasa con la persona, e incluso la más cercana. Pero ¿dónde queda la relación?, y ¿cómo es la relación? Algunas de estas deficiencias prácticas pudieran ser causadas por:

- Carencia de una supervisión y evaluación objetiva de la calidad de la relación y su técnica, debido a la ausencia de datos necesarios para su establecimiento en los protocolos de acogida de los distintos centros hospitalarios.

- Las contra-transferencias que tienen lugar en la relación con la otra persona no son supervisadas $\mathrm{y}$, como consecuencia, no drenadas a nivel organizativo ni a nivel estructural, dando lugar a prejuicios por parte de las enfermeras para relacionarse con la persona.

- Hay fallos en una relación de ayuda estructurada y programada hacia el paciente, la familia y su entorno.

- Falta de compresión y aceptación por parte del paciente y de la manera de manifestar sus necesidades y su entendimiento de la enfermedad, especialmente cuando la persona procede de un entorno cultural diferente al imperante.

- Existencia de tensión profesional a causa de la acumulación de tareas y de falta de identidad profesional en relación con otras profesiones.

Colliere, según Medina (7), en el libro La pedagogía del cuidado, menciona que las decisiones que comprenden acompañamiento, ayuda y colaboración para satisfacer las necesidades básicas, el estímulo para el desarrollo y la autonomía respecto de la enfermedad, cuidados todos ellos vitales, han sido, a partir del siglo XIX, abandonados a la entrega, a la conciencia profesional, a la obligación de toda buena enfermera, convirtiéndose en acciones de segunda categoría, en tareas que no requieren especial pericia. $\mathrm{O}$ bien, cuando una enfermera los prodiga -como ayudar a un enfermo a expresar o a recuperar el deseo de vestirse- no los considera como parte de su trabajo, como actividades propias que exigen un gran discernimiento fruto de la reflexión y de una utilización de conocimientos diversificados.

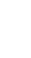


Conviene recordar aquí que la relación terapéutica es la interacción paciente-profesional de enfermería con el propósito de ayudar a los pacientes a que vayan tomando decisiones que mejoren su estilo de vida.

\section{La relación de ayuda}

Esta relación es "aquella que tiene como objetivo ayudar a personas enfermas o con problemas a constatar y ver que hay diferentes maneras de hacer frente a una misma situación o problema, diferentes formas de ver una misma realidad, diferentes caminos para llegar al mismo lugar... es también la creación de relaciones humanas que potencien actitudes facilitadoras para la interacción, nos va a permitir trabajar en los cuidados, prevenir, ayudar a la persona a movilizar sus recursos, nos va a permitir crear condiciones favorables para el desarrollo de la persona, de la familia o del grupo... y va a favorecer nuestro crecimiento personal" (6).

La relación de ayuda no se da entre dos semejantes sino que se da entre dos personas que se encuentran en dos niveles y roles muy distintos. Cibanal y Arce (6) determinan 17 requisitos que se deben tener en cuenta en toda relación de ayuda, orientados hacia la empatía, la autenticidad, la capacidad de escucha y el evitar la minimización o dramatización.

El capítulo "Las fases de la relación de ayuda" describe las etapas a seguir en este proceso, con base en las propuestas de H. Peplau. Cibanal y Arce (6) las remodelan y explican así:

1. "Configuración del encuentro personal: acogida y orientación. Esta fase se refiere a la recepción adecuada del paciente tanto en el ámbito físico como psicológico. Así, en el ámbito físico se dan las características que debe tener el espacio fisico, tales como el aspecto, expresión, etc., que debe tener el profesional de la salud para mostrar una sensación de acogida al paciente. En el ámbito psicológico se vuelve a hacer hincapié en la empatía y la disponibilidad, entre otros aspectos que debe mostrar el enfermero.

2. Exposición, clarificación e identificación del problema: el objetivo último de esta fase es definir el problema. Para ello, también es necesario que el profesional posea determinadas actitudes como las ya repetidas de la escucha activa, empatía, autenticidad y respeto, además de saber ayudar al paciente a concretarse en su problema, a comprometerse y responsabilizarse del trabajo terapéutico, etc. Los autores muestran ejemplos de conversaciones que pueden ayudar a guiar la entrevista. En esta parte también se analizan las actitudes tanto positivas como negativas que puede mostrar el paciente durante esta fase.

3. Confrontación y reestructuración del problema. En esta fase el enfermero debe ayudar al paciente a cambiar su visión del problema y considerar, en esta nueva visión, distintas perspectivas de solución. Adicionalmente, se debe concienciar al paciente de que él es el responsable de sus experiencias y vivencias, se le debe enseñar a aceptar que existen problemas que no se pueden solucionar, etc. También se explican las actitudes que, tanto el enfermero como el paciente, deben tener o evitar en esta fase.

4. Iniciación del plan de acción: decisión del diagnóstico, de los objetivos y plan de acción.

5. El fin de la relación o separación: para la mayoría de profesionales de enfermería no es fácil decir adiós a una persona a la que se debe haber tomado cariño después de una relación de ayuda. Es este apartado se tratan las actitudes que es necesario tener en cuenta para consolidar la obra que se ha realizado, mediante la autonomía del individuo.

6. La evaluación: debe implicar tanto una autoevaluación como una evaluación del proceso completo: ¿se cumplieron los objetivos?" (6).

La conservación de un papel profesional es esencial para trabajar con los pacientes. Un paciente llega a una institución de salud en busca de ayuda y no para obtener relaciones o interacciones de tipo social. El paciente necesita una (un) enfermera(o) y no una amiga (o). Por ello, no resulta conveniente simpatizar durante la relación terapéutica; como el ambiente terapéutico es de tipo social, el profesional de enfermería no tiene que ofrecer información personal de sus metas, intereses o problemas a los pacientes.

La relación enfermera(o)-paciente es un aspecto muy importante para desarrollar los cuidados de enfermería. Es necesario establecer una relación basada en la confianza como requisito indispensable para la buena marcha del proceso terapéutico. No sólo el enfermo debe confiar en la (el) enfermera(o), también ésta(e) debe confiar en sí misma(o) como terapeuta, en la motivación del paciente y en las capacidades para su cambio. 
La confianza es la base fundamental de una relación terapéutica, razón por la cual la consistencia y el establecimiento de límites se constituyen en los elementos claves para generarla. Los límites efectivos proporcionan sentido a la relación establecida y reducen al mínimo la manipulación del paciente.

\section{Responsabilidades}

- Proporcionar supervisión y autocuidado al paciente hasta que llegue el momento en que pueda conservar su autocuidado.

- Cooperar con otros profesionales para garantizar un ambiente terapéutico que contribuya en la recuperación del paciente.

- Proporcionar retroalimentación al paciente con base en las observaciones de su evolución.

- Motivar la participación del paciente y sus cuidadores en el desarrollo de acciones que contribuyan a la recuperación.

\section{Características de los cuidadores eficaces}

El acto de cuidar es propio de la naturaleza humana, de modo que todo ser humano es capaz de cuidar de sí y de otro. El objetivo del trabajo de enfermería es el cuidado, premisa que ha sido aceptada por los profesionales y que promueve que, cuando se define enfermería, se diga que ésta es la ciencia y el arte del cuidado.

El cuidado del individuo, la familia y la comunidad constituyen el centro de atención y el deber ineludible de cualquier profesional de enfermería. La calidad de este cuidado requiere de personas con habilidades interpersonales que brinden cuidados diferenciales y significativos a quien los recibe.

En este sentido, existen habilidades de mayor importancia para el profesional de enfermería que brinda atención y cuidado a personas con trastornos mentales, las cuales hacen referencia a la consideración positiva, el abordaje de no juzgar, la empatía y la paciencia:

\section{Consideración positiva}

Hace referencia a la preocupación, respeto, interés y actitud que demuestra el profesional de enfermería por la situación actual que vivencia el paciente; es creer en el valor y potencialidades que tiene la persona con alteraciones mentales.
La consideración positiva la demuestra la (el) enfermera(o) mediante el desarrollo de acciones que brinden respuesta a las necesidades sentidas por el paciente y no en las que considere importante la (el) enfermera(o).

El profesional de enfermería debe brindar al paciente la oportunidad de expresar sus sentimientos, generalmente inhibidos, para facilitar su autoaceptación. Para ello, le acepta sin condiciones de valor y evita corregirle o dirigirle hacia criterios preestablecidos (8)

\section{Abordaje de no juzgar}

Significa que las (los) enfermeras(os) no realizan críticas severas del comportamiento de los pacientes, permiten que los pacientes hablen de sus pensamientos y sentimientos, respetándolos como seres humanos responsables y capaces de tomar decisiones.

El profesional de enfermería acepta a cada paciente con sus fortalezas y debilidades, preocupándose por ayudarle a entender la situación actual que vivencia y su propia personalidad.

Las (los) enfermeras(os) eficaces aceptan la conducta del paciente siempre y cuando no vaya en detrimento de él o de quienes lo rodean; siempre valoran las probables consecuencias de la conducta del paciente, formulando intervenciones que estén de acuerdo con la situación.

\section{Empatía}

Se define como la habilidad cognitiva que es inherente a un individuo para tomar la perspectiva del otro o de entender algunas de sus estructuras de mundo, sin adoptar necesariamente esta misma perspectiva; es como ponerse en los zapatos del otro y, aunque no se piensa igual, se conoce o se entiende lo que ese otro siente, a sabiendas de que cada individuo posee un guion propio (8). La empatía es una estrategia necesaria para iniciar una buena relación terapéutica.

La (el) enfermera(o) debe dar señales de que se pone en el lugar del enfermo y comprende sus sentimientos, aunque a veces el paciente se muestre agresivo, irritado, nervioso o se encierre en sí mismo. Detrás del comportamiento del paciente siempre hay una razón, un miedo, una frustración o una dificultad, razones por las cuales es fundamental preguntar por los motivos de estos comportamientos. 
ISSN 1794-9831 E-ISSN 2322-7028 Vol. 12 No. 1 Ene - Jun 2015 Cúcuta, Colombia
La empatía se produce sólo si las (los) enfermeras(os) son capaces de comunicar verbalmente lo que entienden para que los pacientes sean capaces de validar o corregir las percepciones de los profesionales de enfermería (9).

En resumen, se puede decir que la empatía no es otra cosa que la habilidad para estar consciente de reconocer, comprender y apreciar los sentimientos de los demás.

\section{Paciencia}

La paciencia es el valor que hace a las personas tolerar, comprender, padecer y soportar los contratiempos y las adversidades con fortaleza, sin lamentarse, moderando sus palabras y su conducta para actuar de manera asertiva frente a cada situación (8). Indica una escucha activa y una propuesta de acciones que estén acordes con las necesidades del paciente, de tal manera que se permita adoptar cambios según lo que se piensa y se planifica. 


\section{REFERENCIAS BIBLIOGRÁFICAS}

1. Páez-Moreno R. La ética de las virtudes y enfermería. Revista Mexicana de Enfermería Cardiológico. 2000; 8(1-4):60-68.

2. Bermedo JC. Humanizar la salud. Rev Enfermer Española 1999; 12(1):42-6.

3. Hernández-Herrera DE, Flores-Hernández S. Relación de ayuda: intervención de enfermería para padres de RN hospitalizado en la UCIN. Revista de Enfermería IMSS 2002; 10 (3): 125-129.

4. Marriner-Tomey A, Raile-Alligood M. Modelos y teorías en enfermería. $4^{a}$ ed. Madrid-España: Editorial Elsevier- Mosb.; 1999.

5. García-Marco MI, López-Ibort MN, Vicente-Edo MJ. Reflexiones en torno a la relación terapéutica: ¿Falta de tiempo? Index Enferm. [Internet]. 2004 [consultado 08 de septiembre de 2005]; 13(47):44-48. Disponible en: $\mathrm{http} / /$ scielo.isciii.es/scielo.php?script=sci_arttext\&pid=S1132-12962004000300010\&lng=es\&nrm=iso

6. Cibanal-Juan ML, Arce MC. La relación enfermera paciente. Medellín: Editorial Universidad de Antioquia; 2009.

7. Medina JL. La pedagogía del cuidado: saberes y prácticas en la formación universitaria en enfermería. Vol. 90. Barcelona - España; Laertes; 1999.

8. Ruiz-Sánchez JJ, Sánchez-Cano JJ. Las Psicoterapias. Introducción a las orientaciones psicoterapéuticas para profesionales sanitarios. www.es.scribd.com [Internet]. Ubeda; 1998 [consultado 08 de septiembre de 2005]. Disponible en: http://es.scribd.com/doc/21196601/LAS-PSICOTERAPIAS\#scribd

9. Sue-Cook J, Lee-Fontaine K. Enfermería psiquiatrita. Fundamentos esenciales de enfermería en salud mental. $2^{\text {a }}$ ed. España: Interamericana Mc-Graw-Hill; 1993. 